\title{
Geographical variation of the alleles at the two prolamin loci, Pro1 and Pro2, in foxtail millet, Setaria italica (L.) P. Beauv.
}

\author{
Hiroki Nakayama ${ }^{1,2}$ * Hyoji Namai $^{2}$ and Kazutoshi Okuno ${ }^{1}$ \\ ${ }^{1}$ National Institute of Agrobiological Resources, Kannondai 2-1-2, \\ Tsukuba, Ibaraki 305-8602, Japan \\ ${ }^{2}$ Institute of Agriculture and Forestry, University of Tsukuba, \\ Tennodai 1-1-1, Tsukuba, Ibaraki 305-8572, Japan
}

(Received 5 August 1999, accepted 15 December 1999)

\begin{abstract}
Allelic variation at the two prolamin loci (Pro1 and Pro2) and its geographical distribution in 560 local cultivars of foxtail millet (Setaria italica) mainly from Eurasia were studied using SDS-polyacrylamide gel electrophoresis (SDSPAGE). Genetic analysis of a newly detected polymorphic band, band 6, indicated that it is controlled by an allele at the Pro2 locus, which was designated as Pro2f. Two alleles (Pro1a and Pro1null) at the Pro1 locus and six alleles (Pro2a, Pro2b, Pro2c, Pro2d, Pro2e and Pro2f) at the Pro2 locus were detected among the cultivars examined. Although the frequency of the Pro1a allele varied from 0\% in the Nansei islands of Japan and Africa to 66\% in Afghanistan, no apparent trend was observed in geographical distribution. In contrast, two common alleles at the Pro2 locus, Pro2b and Pro2f, had clear differential geographical distribution. The Pro2b allele was most frequent in Europe and decreased in frequency eastwards. The Pro2f allele occurred frequently in subtropical and tropical regions including the Nansei islands of Japan, the Philippines, Nepal, India, Pakistan and Africa. All eight alleles at the Pro1 and Pro2 loci occurred in China, suggesting China is a center of diversity. The origin of geographical differentiation of local cultivars into a "tropical group" characterized by the Pro $2 f$ allele and other genes was discussed.
\end{abstract}

\section{INTRODUCTION}

Foxtail millet, Setaria italica (L.) P. Beauv. is an annual crop, which is mainly grown in the temperate zone of Eurasia and shows a wide range of phenotypic variation (Nguyen Van and Pernes, 1985; Takei and Sakamoto, 1987; Li et al., 1995). However, there have been few studies concerning the genetic diversity among local cultivars based on the polymorphism of biochemical and molecular markers (Jusuf and Pernes, 1985; Fukunaga et al., 1997)

Prolamin, an alcohol soluble storage protein in cereal seeds, has been widely used as a biochemical marker to clarify intraspecific variation because they are not greatly affected by natural selection and are highly polymorphic (Ciaffi et al., 1993; Metakovsky and Branlard, 1998; Portyanko et al., 1998). In foxtail millet, prolamin is a major component of seed storage protein in endosperm and its variation in SDS-PAGE electrophoregram comprises the combination of five polymorphic bands (bands $1-5$; see

\footnotetext{
* Corresponding author.
}

Fig. 1). The genetic analysis involving these bands showed that band 3 is governed by an allele at the Pro1 locus, whereas bands 1, 2, 4 and 5 are controlled by five alleles at the Pro2 locus, which is not linked to Pro1 (Nakayama et al., 1999). Gene diversity (Nei, 1973) based on the frequency of alleles at these two loci was apparently comparable to that of isozymes, suggesting it is a potentially useful genetic marker. In the further analysis of additional cultivars using alternative procedures for SDS-PAGE, a new polymorphic band, tentatively designated as band 6, was detected in cultivars from Southeast Asia and South Asia at a high frequency. The objectives of the present study were to investigate the genetic control of band 6 and to assess geographical variation of foxtail millet based on allelic variation at the two prolamin loci, Pro1 and Pro2.

\section{MATERIALS AND METHODS}

For the genetic analysis of a new prolamin band (band 6 ; see Fig. 1), one hundred and sixty two $\mathrm{F}_{2}$ seeds derived 
from the cross between a Japanese cultivar (collection number K74-10-4-4; with bands 4 and 5) and a Philippine cultivar (collection number 77-10-7-25; with bands 4 and 6) were analyzed. The parental cultivars were originally introduced from the Plant Germ-plasm Institute, Kyoto University and have been maintained at the Laboratory of Plant Genetic Diversity, National Institute of Agrobiological Resources. A total of 560 local cultivars originating mainly from Eurasia were used to investigate the geographical variation of alleles at the two prolamin loci (Table 1). The analysis was repeated at least twice to ensure homogeneity within each cultivar.

The meal of a single dehulled seed prepared from each cultivar and each $\mathrm{F}_{2}$ seed was placed in a $1.5 \mathrm{ml}$ tube and prolamin was extracted with $70 \%$ (v/v) aqueous ethanol. The precipitate was recovered by evaporation of aqueous ethanol and was redissolved with $50 \mu \mathrm{l}$ of buffer solution: $0.05 \mathrm{M}$ Tris-HCl buffer ( $\mathrm{pH}$ 8.0) containing $0.2 \%$ SDS, $1 \%$ of 2-mercaptoethanol and $5 \mathrm{M}$ urea. Ten to $15 \mu \mathrm{l}$ of each prolamin sample was analyzed by SDS polyacrylamide gel
(kDa) (a)

29.0

20.1

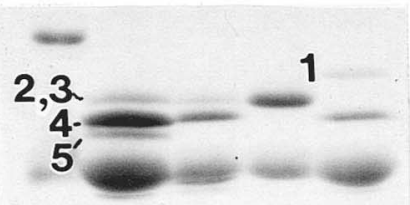

14.2 (b)

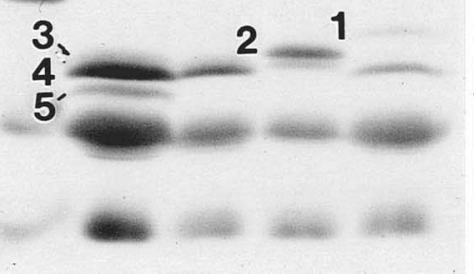

$\begin{array}{llll}5 & 6 & 7 & 8\end{array}$ (c)
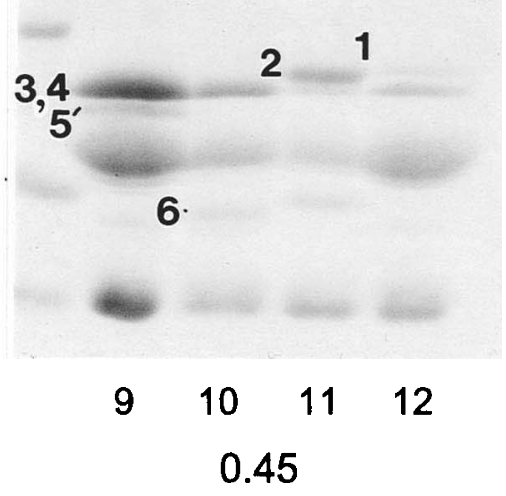

$0.25 \mathrm{M}$

\section{Tris in separation gel}

2.6

$0.75 \mathrm{M}$

$0.75 \mathrm{M}$

Fig. 1. Effects of the degree of cross-linking (C) and concentration of Tris on the resolution of prolamin bands. The nomenclature of the bands is after Nakayama et al. (1999). Lane 1, 5 and 9, 80-10-9-5-1 (collection number of Kyoto University) bearing bands 3, 4 and 5; lane 2, 6 and 10, 77-10-7-2 bearing bands 3,4 and 6 ; lane 3,7 and 11, 81-6-10-1 bearing bands 2 and 3; lane 4, 8 and 12, 7811-14-14 bearing bands 1 and 4, respectively.

${ }^{a}$ Degree of cross-linking $\mathrm{C}=b /(a+b)(\%)$,

$a$ is the mass of acrylamide and $b$ is the mass of methylenebisacrylamide (BIS), respectively.

Table 1. Frequency (\%) of the alleles at the two prolamin loci

\begin{tabular}{|c|c|c|c|c|c|c|c|c|c|c|}
\hline \multirow[b]{3}{*}{ Origin } & \multirow{2}{*}{$\begin{array}{r}\text { Loci } \\
\text { Alleles }\end{array}$} & \multicolumn{2}{|c|}{ Pro1 } & \multirow[b]{2}{*}{$a$} & \multirow[b]{2}{*}{$b$} & \multicolumn{2}{|c|}{ Pro2 } & \multirow[b]{2}{*}{$e$} & \multirow[b]{2}{*}{$f$} & \multirow[b]{3}{*}{$H^{\mathrm{c}}$} \\
\hline & & $a$ & mull & & & $c$ & $d$ & & & \\
\hline & cultivars & $3^{\mathrm{a}}$ & - & 4 & $4+5$ & $1+4$ & 2 & $2+5$ & $4+6$ & \\
\hline Japan $^{\mathrm{b}}$ and Korea & 65 & 6 & 94 & 82 & 3 & 9 & 2 & 0 & 5 & 0.219 \\
\hline China & 150 & 25 & 75 & 65 & 13 & 13 & 1 & 1 & 9 & 0.455 \\
\hline Taiwan & 36 & 28 & 72 & 92 & 3 & 0 & 0 & 0 & 6 & 0.279 \\
\hline The Nansei islands (Japan) & 23 & 0 & 100 & 48 & 0 & 0 & 0 & 0 & 52 & 0.250 \\
\hline The Philippines & 17 & 59 & 41 & 41 & 0 & 0 & 0 & 0 & 59 & 0.484 \\
\hline Nepal & 37 & 30 & 70 & 59 & 8 & 0 & 0 & 0 & 32 & 0.476 \\
\hline India & 45 & 18 & 82 & 47 & 18 & 0 & 0 & 0 & 36 & 0.458 \\
\hline Pakistan & 41 & 46 & 54 & 59 & 5 & 0 & 5 & 0 & 32 & 0.525 \\
\hline Afghanistan & 41 & 66 & 34 & 100 & 0 & 0 & 0 & 0 & 0 & 0.225 \\
\hline Iran, Central Asia and Turkey & 35 & 6 & 94 & 74 & 23 & 0 & 0 & 0 & 3 & 0.251 \\
\hline Europe (including CIS) & 58 & 31 & 69 & 55 & 40 & 5 & 0 & 0 & 0 & 0.482 \\
\hline Africa & 12 & 0 & 100 & 50 & 0 & 8 & 0 & 0 & 42 & 0.285 \\
\hline Total & 560 & 26 & 74 & 67 & 12 & 5 & 1 & $<1$ & 16 & 0.450 \\
\hline
\end{tabular}

${ }^{a}$ Prolamin bands controlled by each allele, see Fig. 2 .

${ }^{\mathrm{b}}$ Except for the Nansei islands

${ }^{\mathrm{c}}$ Gene diversity of Nei (1973) given by the mean value of $h$ for the Pro1 and Pro2 loci.

$h=1-\sum_{i=1}^{m} P i^{2}$, where $m$ and $P i$ are number of alleles and the frequency of $i$ th allele, respectively. 
electrophoresis (SDS-PAGE) using the discontinuous buffer system based on Laemmli (1970). Previous study showed that the resolution of proteins in SDS-PAGE electrophoregrams could be modified by either changing the degree of cross-linking $(\mathrm{C})$, which is the percentage of the mass of methylenebisacrylamide (BIS) to the sum of the mass of BIS and that of acrylamide in a separation gel (Kagawa et al., 1988), or changing the concentration of Tris (hydroxymethylaminomethane) in a separation gel (Okajima et al., 1993). Accordingly, three types of separation gel having different $\mathrm{C}$ and different Tris concentrations were used to obtain best resolution for each band. They are separation gels having $2.6 \%$ of $\mathrm{C}$ containing $0.75 \mathrm{M}$ Tris (Fig. 1a), those having $0.45 \%$ of $\mathrm{C}$ containing $0.75 \mathrm{M}$ Tris (Fig. $1 \mathrm{~b}$ ), and those having $0.45 \%$ of $\mathrm{C}$ containing $0.25 \mathrm{M}$ Tris (Fig. 1c).

\section{RESULTS AND DISCUSSION}

Detection and genetic analysis of band 6. Four polymorphic bands (bands 1, 4, 5 and, 2 or 3 of the same mobility) were observed in the range of 20 to $30 \mathrm{kDa}$ using a separation gel having $2.6 \%$ of the degree of cross-linking (C) (Fig. 1a) containing $0.75 \mathrm{M}$ Tris. Bands 2 and 3 could not be separated under these conditions because they showed identical mobility. By changing $\mathrm{C}$ from 2.6 to 0.45 , bands 2 and 3 could be separated. Accordingly, this

Table 2. Segregation for prolamin bands 5 and $6 \mathrm{~F}_{2}$ seeds of the cross between K74-10-4-4 (with band 4 and 5) and $77-10-7-25$ (with band 4 and 6 )

\begin{tabular}{|c|c|c|c|c|c|c|}
\hline a) Bands & $+^{\mathrm{a}}$ & - & & Total & $\begin{array}{l}\chi^{2} \text { for } \\
3: 1\end{array}$ & $\mathrm{P}$ \\
\hline 5 & 118 & 44 & & 162 & 0.403 & $0.75-0.50$ \\
\hline 6 & 116 & 46 & & 162 & 0.996 & $0.50-0.20$ \\
\hline b) Bands & $+1-$ & $+1+$ & $-1+$ & Total & $\begin{array}{c}\chi^{2} \text { for } \\
1: 2: 1\end{array}$ & $\mathrm{P}$ \\
\hline $5 / 6$ & 46 & 72 & 44 & 162 & 2.049 & $0.50-0.20$ \\
\hline
\end{tabular}

a + : present -: absent modification was complimentarily used for the analysis involving bands 2 and 3 (Fig. 1b). Additionally, further analysis using separation gels having $0.45 \%$ of $\mathrm{C}$ containing $0.25 \mathrm{M}$ of Tris, a new polymorphic band, band 6 was detected between $14 \mathrm{kDa}$ and $20 \mathrm{kDa}$ (Fig. 1c). The band 6 frequently occurred among the cultivars from the subtropical and tropical regions ranging from the Nansei islands of Japan to Pakistan.

To examine the genetic control of band 6 , the $\mathrm{F}_{2}$ seeds derived from a cross between a Philippine cultivar (with bands 4 and 6) and a Japanese cultivar (with bands 4 and 5) were analyzed. Bands 5 and 6 segregated into the ratio of 3 (presence):1 (absence) (Table 2a). This indicates that each of the prolamin bands is controlled by a single dominant gene. Segregation for both bands 5 and 6 together fitted the ratio of 1:2:1 (Table 2b), which shows that the genes controlling these bands are allelic. The genetic analysis involving five prolamin bands 1, 2, 3, 4 and 5 previously showed that band 3 is governed by an allele at the Pro1 locus, whereas bands 1, 2, 4 and 5 are controlled by alleles at the Pro2 locus, which is not linked to the Pro1 locus (Nakayama et al, 1999). In the previous result, two alleles at the Pro1 locus, Pro1a (controlling band 3) and Pro1null, and five alleles at the Pro2 locus, Pro2a (band 4), Pro2b (bands 4 and 5), Pro2c (bands 1 and 4), Pro2d (band 2) and Pro2e (bands 2 and 5) were detected among 271 local cultivars from various regions of Eurasia. Since bands 5 and 6 are allelic, it can be concluded that band 6 is also controlled by an allele at the Pro2 locus and the allele controlling bands 4 and 6 in the Philippine cultivar is newly designated as Pro2f (Fig. 2).

\section{Geographical distribution of alleles at the Pro1 and} Pro2 loci. Among 560 local cultivars examined, two alleles at the Pro1 locus and six alleles at the Pro2 locus were detected (Fig. 2). Pro1null at the Pro1 locus occurred in $74 \%$ of total cultivars (Table 1). For the Pro2 locus, $67 \%$ of the cultivars bore Pro2 $a$ and $16 \%$ and $12 \%$ of the remaining cultivars had Pro2f and Pro2b, respectively. The other three alleles (Pro2c, Pro2d and Pro2e) were rare,

\section{Locus}

\section{Pro1}

Band no.

3

\section{Pro2}

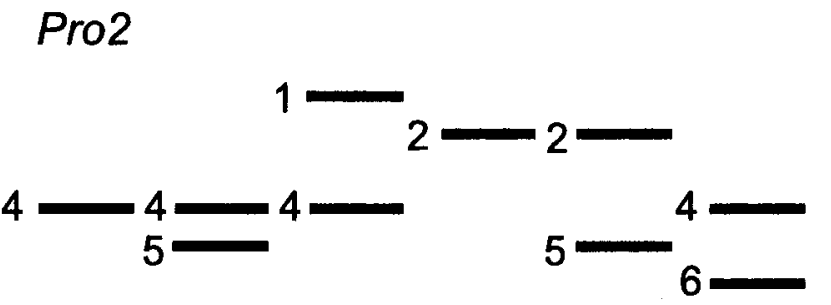

\section{Alleles Pro1a Pro1null Pro2a Pro2b Pro2c Pro2d Pro2e Pro2f}

Fig. 2. Allelic variation at the two prolamin loci, Pro1 and Pro 2 in foxtail millet. 
accounting for less than $7 \%$ of cultivars. Eleven out of twelve possible genotypes based on the combination of alleles at the two prolamin loci were found among cultivars and no particular pattern of association was found between alleles at the two loci.

Frequency of the Pro1a allele at the Pro1 locus varied from $0 \%$ in the Nansei islands of Japan and Africa to $66 \%$ in Afghanistan, followed by $59 \%$ in the Philippines (Table 1). Despite the variation in frequencies among regions, Pro1a distributed in almost all the regions and no apparent trend was observed in geographical distribution.

Frequency of each allele at the Pro2 locus differed among regions (Table 1; Fig. 3). The Pro2a allele was commonly detected in cultivars throughout Eurasia. Geographical distribution of two other frequent alleles, Pro2b and Pro2f showed different trends. The Pro $2 b$ allele was the most frequent in Europe. The frequency of this allele decreased eastwards and was rare in Japan and Korea, Taiwan, Nepal and Pakistan. The Pro2f allele occurred frequently in subtropical and tropical regions including the Nansei islands of Japan, the Philippines, Nepal, India, Pakistan and Africa, and was uncommon in other regions. The distribution of two rare alleles, Pro2c and Pro2d was sporadic. Pro2c occurred frequently in East Asian countries such as China, Japan and Korea, and in Europe. One cultivar from Morocco in north Africa also bore Pro2c. Pro2d was detected in cultivars from Japan and Korea, China and Pakistan at a low frequency. One cultivar with Pro2e, possibly derived from the intralocus recombination between Pro $2 b$ and Pro2d, was only found in one Chinese cultivar.

The gene diversity (Nei, 1973) calculated from the frequency of alleles at the two prolamin loci varied from 0.219 in Japan and Korea to 0.525 in Pakistan (Table1). There are several regions with high level of diversity, such as China, the Philippines, Nepal, India, Pakistan and Europe and no distinct center of diversity was identified. On the other hand, the number of alleles at the Pro1 and Pro2 loci indicated that China is the most diverse region for prolamin alleles, since all eight alleles were detected among the cultivars from China (Table 1). The high level of gene diversity in China was derived both from a large number of alleles and their even frequency, whereas those in other regions were solely due to their evenness of allelic frequency in spite of a fewer number of alleles at the two loci. Judging from the abundance of prolamin alleles as well as the gene diversity, it is possible to consider China is a center of diversity of foxtail millet. This hypothesis is in accordance with Vavilov (1926) who stated that the East Asian countries including China and Japan were the principle center of diversity of foxtail millet. Cluster analysis of morphological characters in foxtail millet also supports this view (Li et al., 1995).

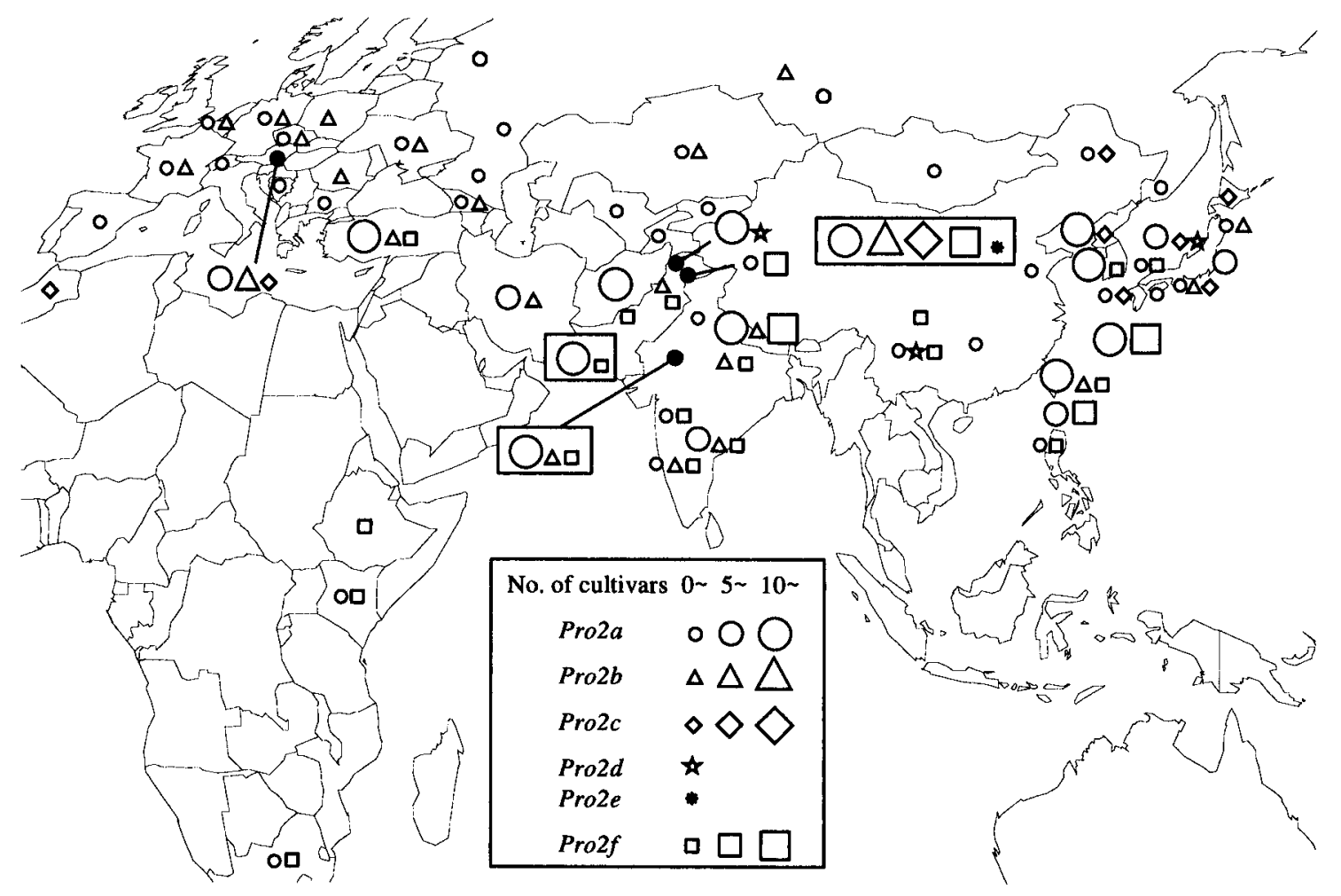

Fig. 3. Geographical distribution of alleles at the Pro2 locus. The cultivars for which only the general geographical origin are known are shown in the center of the territories or in the box. 
Origin of the genetic differentiation of tropical cultivars. The Pro2f allele occurred mainly in subtropical and tropical regions ranging from the Nansei islands of Japan to Pakistan (Table 1). These are the regions where cultivar with phenol color reaction prevailed (Kawase, 1986) and type II and III of rDNA RFLP pattern are mainly found (Fukunaga et al, 1997). Furthermore, the geographic variation of alleles at ten isozyme loci indicated that the cultivars from these regions were characterized by the occurrence of $P g d 2 b$ or $P g d 2 c$ alleles at a high frequency (Jusuf and Pernes, 1985). Since the subtropical and tropical cultivars share these specific alleles and phenotypes in common, the genetic differentiation of subtropical and tropical cultivars into a "tropical group" is strongly suggested. Genetic differentiation of Asian cultivars into temperate and tropical groups has also been shown by RAPD analysis (Schontz and Rether, 1999).

The region where foxtail millet was domesticated and routes of dissemination have not been determined. However, since most of the alleles and phenotypes which characterize the tropical group also exist in Chinese cultivars, it is possible that the tropical group originated from Chinese cultivars. In addition, some characters such as white anther and glutinous endosperm which are not present in Setaria viridis, the wild ancestor of foxtail millet, are found in Chinese and tropical cultivars (Nakayama, unpublished data). This implies that the genetic differentiation occurred after domestication of foxtail millet from Setaria viridis. Thus it seems likely that the tropical group originated from some Chinese cultivars which migrated to Southeast Asia and South Asia and differentiation followed as a result of geographical isolation.

We would like to thank Dr. M. Kawase, Shikoku National Agricultural Experiment Station, Japan, Dr. K. Fukunaga, Okayama University, Japan, Dr. M. Sato, Kagoshima University, Japan, and North Central Regional Plant Introduction Station, USDAARS for providing the local cultivars of foxtail millet from various countries used in the experiments. We greatly appreciate Dr. R. Osawa, University of Tsukuba, Dr. H. Morishima and Dr. D. A. Vaughan of National Institute of Agrobiological Resources, Japan for their critical reading and advices.

\section{REFERENCES}

Ciaffi, M., Lafiandra, D., Porceddu, E., and Benedettelli, S. (1993)
Storage-protein variation in wild emmer wheat (Triticum turgidum ssp. dicoccoides) from Jordan and Turkey. II. Patterns of allele distribution. Theor. Appl. Genet. 86, 518-525.

Fukunaga, K., Domon, E., and Kawase, M. (1997) Ribosomal DNA variation in foxtail millet, Setaria italica (L.) P. Beauv., and a survey of variation from Europe and Asia. Theor. Appl. Genet. 95, 751-756.

Jusuf, M., and Pernes, J. (1985) Genetic variability of foxtail millet (Setaria italica P. Beauv.). Theor. Appl. Genet. 71, 385391.

Kagawa, H., Hirano, H., and Kikuchi, F. (1988) Variation of glutelin seed storage protein in rice (Oryza sativa L.). Japan. J. Breed. 38, 327-332.

Kawase, M. (1986) Genetic variation and landrace differentiation of foxtail millet, Setaria italica, in Eurasia. Ph. D. thesis, Kyoto University, Japan.

Laemmli, U. K. (1970) Cleavage of structural proteins during the assembly of the head of bacteriophage T4. Nature 227, 680685.

Li, Y., Wu, S., and Cao, Y., (1995) Cluster analysis of an international collection of foxtail millet (Setaria italica (L.) P. Beauv.). Euphytica 83, 79-85.

Metakovsky, E. V., and Branlard, G. (1998) Genetic diversity of French common wheat germplasm based on gliadin alleles. Theor. Appl. Genet. 96, 209-218.

Nakayama, H., Namai, H., and Okuno, K. (1999) Genes controlling prolamin biosynthesis, Pro1 and Pro2, in foxtail millet, Setaria italica (L.) Beauv. Genes Genet. Syst. 74, 93-97.

Nei, M. (1973) Analysis of gene diversity in subdivided populations. Proc. Nat. Acad. Sci. 70, 3321-3323.

Nguyen Van, E., and Pernes, J. (1985) Genetic diversity of foxtail millet (Setaria italica). In: Genetic Differentiation and Dispersal in Plants (eds: P. Jacquard et al. ), pp. 113-128. NATO ASI series, Vol. G5, Springer-Verlag, Berlin Heidelberg.

Okajima, T., Tanabe, T., and Yasuda, T. (1993) Nonurea sodium dodecyl sulfate-polyacrylamide gel electrophoresis with highmolarity buffers for the separation of proteins and peptides. Anal. Biochem. 211, 293-300.

Portyanko, V. A., Sharopova, N. R., and Sozinov, A. A. (1998) Characterisation of European oat germ plasm: allelic variation at complex avenin loci detected by acid polyacrylamide gel electrophoresis. Euphytica 102, 15-27.

Schontz, D., and Rether, B. (1999) Genetic variability in foxtail millet, Setaria italica (L.) P. Beauv.: Identification and classification of lines with RAPD markers. Plant Breed. 118, 190-192.

Takei, E., and Sakamoto, S. (1987) Geographical variation of heading response to daylength in foxtail millet (Setaria italica P. Beauv.). Japan. J. Breed. 37, 150-158.

Vavilov, N.I. (1926). Studies on the Origin of Cultivated Plants, pp248. Inst. Appl. Bot. Plant Breed., Leningrad. 